\title{
COMMUNICATION
}

\section{Fistules cutanées d'origine dentaire : Deux cas cliniques}

\section{Taihi-Nassif I $\left.\right|^{\star 1,2}$, Danièle Guemkam D *1,2, Ben Lagha N}

${ }^{*}$ Co-auteurs

1 - Service de stomatologie, Hôpital André Grégoire, Montreuil 93300

2 - Faculté de chirurgie dentaire de Paris Descartes, Montrouge 92120

\section{Introduction}

Les fistules sont le signe de diffusion d'un processus infectieux chronique non traité. Au niveau cervico-facial, elles siègent principalement au niveau des muqueuses et sont rares dans la peau. Les infections d'origine dentaire peuvent donner des fistules cutanées. Souvent méconnues par les praticiens et confondues avec des lésions dermatologiques bénignes, elles amènent le patient à consulter différents spécialistes (dermatologues, ORL,...). 49\% des patients ont déjà subi un traitement médicamenteux ou chirurgical en méconnaissance de l'étiologie, laissant des cicatrices inesthétiques permanentes.

\section{Observations}

La première patiente âgée de 45 ans, en bonne santé générale, adressée pour une lésion cutanée présente depuis plusieurs semaines est réfractaire aux traitements anti-inflammatoires locaux. Elle ne se plaint d'aucune douleur. L'examen exobuccal montre un nodule cutané sous mandibulaire gauche de $1 \mathrm{~cm}$ de diamètre, recouvert d'une croûte. L'examen endobuccal révèle une restauration non adaptée sur 37, la percussion axiale est douloureuse ainsi que la palpation vestibulaire. La panoramique retrouve une image radioclaire infracentimétrique sur 37 . Le diagnostic de fistule cutanée d'origine dentaire est évoqué. L'avulsion de 37 est pratiquée vu son pronostic réservé. Après 3 semaines, la cicatrisation cutanée est quasi-complète avec persistance d'un léger érythème. La patiente est satisfaite et ne souhaite plus recourir à la chirurgie esthétique.

Le deuxième patient est un homme de 21 ans, en bonne santé générale, qui consulte pour un gonflement mentonnier depuis deux mois. L'examen exobuccal montre une tuméfaction sous mentonnière mesurant $1 \mathrm{~cm}$ de diamètre, entourée d'une aréole inflammatoire, souple et non douloureuse à la palpation. L'examen endobuccal révèle un comblement vestibulaire mandibulaire qui s'étend de 31 à 34 , induré à la palpation. La 33 est absente de l'arcade. La panoramique et le scanner mandibulaire révèlent l'inclusion de 33 entourée d'une image radioclaire mesurant $1,5 \mathrm{~cm}$ de diamètre. Le diagnostic de fistule cutanée d'origine dentaire est posé. L'exérèse du kyste folliculaire et de la 33 est réalisée. L'examen anatomo-pathologique confirme la nature du kyste. La cicatrisation cutanée est quasi-complète à 1 mois post opératoire.

Discussion et conclusion

Les infections d'origine dentaire sont les causes les plus fréquentes des fistules cutanées de la face et du cou (Cantatore JL et al. Cutis. 2002 Nov;70(5):264-7). Elles touchent principalement 
les jeunes. La mandibule est touchée dans $87 \%$ des cas, souvent dans l'angle mandibulaire (Guevara-Gutiérrez E et al. Int J Dermatol. 2015 Jan;54(1):50-5). Les formes cliniques sont variables. La forme nodulaire représente $53 \%$ des cas. Leur taille varie de quelques millimètres à deux centimètres. Le diagnostic est difficile vu le peu de symptomatologie douloureuse. II est souvent radiologique, permettant de suivre le trajet de la fistule. Les examens histologiques et microbiologiques sont possibles mais non nécessaires. Le traitement étiologique présente le moyen efficace et rapide à la disparition du préjudice esthétique. II rend le procédé chirurgical inutile (Kumar U et al. BMJ Case Rep. 2014 Jul 15;2014). Ces cas soulignent l'importance de la formation des dermatologues et des chirurgiens-dentistes à ce diagnostic et d'évoquer l'étiologie dentaire devant toute lésion faciale ou cervicale ne répondant pas aux thérapeutiques dermatologiques conventionnelles. 